learn about chosen topics, and present audits or other projects. The loss of these sessions is understandable, but no less frustrating. There has been a recent rise in use of video communication. Apps like Houseparty and Zoom help us socialise from our homes and this idea inspired me to consider other means of group teaching. I therefore arranged a journal club on Microsoft Teams, which took place instead of our usual consultant-led session. We now plan to hold regular video sessions to present and discuss other papers.

The current crisis does not mean that no teaching can happen, but rather that conventional means have to be replaced. Given the severe reduction in learning opportunities for trainees, I am pleased that video meetings are a suitable alternative.

B. Dunphy, Norwich, UK https://doi.org/10.1038/s41415-020-1546-2

\section{Out of our comfort zone}

Sir, during the COVID-19 crisis redeployment of the dental profession is a controversial topic. With the wide variety of skillsets present within our profession, and the passion to help both our patients and the wider healthcare workforce, it is time for the dental profession to step out of our comfort zone in order to use these vital skills to aid the community and our NHS.

It was stated that over 13,000 dental professionals had signed up to volunteer for redeployment, which highlights the profession's willingness to unite with the rest of the NHS workforce to win the fight against COVID. This subject has caused many members of the profession to take to social media to express concerns over job roles, either with regards to a lack of skillset or, in contrast, to the underutilisation of skills present.

We can bring more than just teeth into the world of healthcare: from history taking, triaging, prescribing, cannulation and suturing, to the privilege of providing reassurance and support to worried families and patients. Existing hospital dentists and trainees have already been tasked with cross-cover of medical specialities and with increased training they are leading deployment to areas within their Trusts. Uncertainty and false information in relation to redeployment on social media within the dental profession will not provide the positivity needed.

With many dentists taking the Hippocratic oath upon graduation and living by a set of professional principles and values throughout their career, it is evident that putting 'patients' interests first' will remain a guiding factor for the work that we do. The entire dental workforce should take pride in being part of the NHS healthcare system and when this crisis is over, we should be able to look back as a profession with pride in our collective and positive response.

\section{A. Thomson, P. Chohan, M. J. Ismail, S. Khalid. N. Patel, S. Uppal, Norwich, UK https://doi.org/10.1038/s41415-020-1542-6}

\section{Caution with NSAIDs}

Sir, as aerosol generating procedures (AGPs) are currently under a moratorium due to COVID precautions, dentists are being advised to use analgesia as a pain temporising method much more than in normal dental practice.

Ibuprofen is being recommended at doses of up to $2,400 \mathrm{mg} /$ day and much more extensive use of diclofenac is being advised. Both are likely to be used for much longer periods than the 5-7 days familiar to dental practitioners. ${ }^{1}$ These are NSAID drugs which are safely used by dentists in healthy individuals, but in certain medical conditions it is important that dentists are aware of the serious adverse effects that they can cause. Patients with asthma, renal disease, liver disease, allergy/angioedema, peptic ulcer disease, acute cardiac and stroke risk and cardiac failure are all groups where special caution is required when using NSAID analgesics. They are also not recommended for use into the third trimester of pregnancy. NICE has issued guidance to medical practitioners starting these medicines for longer term or high dose use and dentists would be well advised to look at this guidance until more specific dental focussed information is available. ${ }^{2}$ In many of these risk groups paracetamol is a safer alternative.

Drug interactions are important and dentists should check a patient's existing medication through the BNF/eBNF/BNFC for unfamiliar interactions when considering high dose or high potency NSAID prescribing. Warfarinised patients should not routinely be given an NSAID. New oral anticoagulants, such as apixaban and dabigatran, are more tolerant of NSAID use although some platelet effects may cause mildly prolonged bleeding. High dose ibuprofen should not be used in patients taking aspirin $75 \mathrm{mg} /$ day as it reduces the cardioprotective effect. ${ }^{3}$

Dentists should remember that in these risk patients, paracetamol and codeine (co-codamol $8 / 500$ ) may be preferred to a NSAID and is available over the counter at a pharmacy or as Solpadeine Max (12.8/500). A stronger version of co-codamol (15/500) is available through a GP's prescription as are other opioid medicines. Where the dentist is unsure about providing high dose or long course analgesia, or when prescribing in the risk groups above, it is sensible to contact the patient's GP for advice before issuing the prescription.

A. Crighton, C. McCann, E. Todd, A. Brown, Glasgow, UK

\section{References}

1. Scottish Dental Clinical Effectiveness Programme Management of Acute Dental Problems During COVID19 Pandemic. Available at: http://www.sdcep.org.uk/ published-guidance/acute-dental-problems-covid-19/ (accessed April 2020).

2. NICE. NSAIDs - prescribing issues. Last revised August 2019. Available at: https://cks.nice.org.uk/nsaidsprescribing-issues\#! scenario (accessed April 2020).

3. Gurbel P, Tantry U, Weisman S. A narrative review of the cardiovascular risks associated with concomitant aspirin and NSAID use. J Thromb Thrombolysis 2019; 47: 16-30.

https://doi.org/10.1038/s41415-020-1543-5

\section{Remain positive and proactive}

Sir, at this time of global uncertainty this is a heartfelt letter to remind our dental practitioners of the ways which we can remain positive and proactive. Redeployment is a daunting prospect; however, it will offer opportunities to give back to our communities. Working in a hospital will provide an insight into the challenges faced as the NHS sustains great pressure. We imagine that dentists will take many skills back to practice, having developed their adaptability, initiative and team-working skills in a completely new environment. As a profession, we should be proud to support our hospitals through this unprecedented time.

For our fellow trainees, you may feel that you are disadvantaged with the absence or reduction in clinical activities. To keep ourselves engaged in dentistry, there are plenty of online resources, webinars and CPD to help us develop and stay connected to colleagues. It may also be a good time to review your personal development plan. Remember we are only human; whilst we strive to give to others during this period, it is important to take care of our own physical and mental wellbeing.

Last but not least, there cannot be a better time for reflection. The hustle and bustle of our daily routine has come to a stop. Sometimes this is exactly what we need to take a step back and ask ourselves key questions, pivotal to our growth as clinicians and individuals.

P. Momin, Manchester, S. Mahmood, Bradford https://doi.org/10.1038/s41415-020-1544-4 